\title{
MRI diagnosis of tubal torsion: the whirlpool sign
}

\author{
Anuj Thakral ${ }^{1 *}$, Rajeev Malhotra ${ }^{1}$, Nitika Sobti $^{2}$, Ankita Chandna $^{2}$, Manisha Arora $^{2}$
}

\author{
${ }^{1}$ Department of Radiology, Max Super specialty Hospital, Shalimar Bagh, Delhi, India \\ ${ }^{2}$ Department of Obstetrics \& Gynaecology, Max Super specialty Hospital, Shalimar Bagh, Delhi, India
}

Received: 17 October 2015

Accepted: 14 December 2015

\section{*Correspondence:}

Dr. Anuj Thakral,

E-mail: anujthakral@gmail.com

Copyright: (C) the author(s), publisher and licensee Medip Academy. This is an open-access article distributed under the terms of the Creative Commons Attribution Non-Commercial License, which permits unrestricted non-commercial use, distribution, and reproduction in any medium, provided the original work is properly cited.

\begin{abstract}
Isolated fallopian tube torsion is a rare cause of acute lower abdominal pain with multiple described contributory factors resulting in tubal necrosis and gangrene. While clinical presentation is nonspecific with a wide clinical differential diagnosis, a combination of sonographic or MRI whirlpool sign with a dilated fallopian tube and normal appearing ipsilateral ovary helps make a preoperative diagnosis of tubal torsion. We report a case of a middle aged female who was diagnosed with tubal torsion on preoperative MRI and is the first description of MRI whirlpool sign for diagnosis of tubal torsion from the Indian subcontinent to our knowledge. The whirlpool mass in tubal torsion is much smaller than ovarian torsion and may be less obvious on ultrasound as in present case.
\end{abstract}

Keywords: Fallopian tube, Magnetic resonance imaging, Ultrasonography, Gangrene

\section{INTRODUCTION}

Isolated fallopian tube torsion (without ovarian torsion) is a rarely reported condition as the cause of acute lower abdominal pain. It is encountered in 1 out of $1-1.5$ million women. ${ }^{1,2}$ The first ever report came in 1890 by BlandSutton. ${ }^{3}$ We report the first case of right tubal torsion diagnosed on imaging and confirmed by surgery from the Indian subcontinent.

\section{CASE REPORT}

A middle aged female presented with pain and heaviness in lower abdomen of 3 days duration. The pain was not relieved by oral analgesics. She had history of 2 normal vaginal deliveries with last child birth 20 years back. Vitals were normal except for mild tachycardia (pulse rate $=102$ beats $/ \mathrm{min})$. Per vaginal examination showed fullness of right fornix with associated tenderness. Left vaginal fornix was free while uterus appeared bulky.
Ultrasound pelvis and transvaginal sonography showed an oblong hypoechoic adnexal lesion with internal echoes in the dependent part. The presence of dense internal echoes raised the suspicion of internal hemorrhage. Both ovaries were well visualized separately and showed normal appearance. Color Doppler showed normal vascularity in both ovaries. An obvious twisted vascular pedicle was not visualized on ultrasonography and color Doppler.

MRI Pelvis performed for further evaluation showed a dilated thick walled tubular structure with internal hemorrhagic contents suggestive of hematosalpinx. There was associated coiled appearance of right adnexal vessels with odema and stranding of surrounding fat. Both ovaries showed normal MRI appearance, thus favoring the possibility of right tubal torsion.

With strong radiological evidence of tubal torsion and persistent symptoms of right iliac fossa pain with tenderness, patient was taken up for diagnostic laparoscopy with laparoscopic salpingectomy. It showed 
a right sided hematosalpinx with presence of adhesions with surrounding bowel and right sided lateral pelvic wall. The right fallopian tube was found to be torsed and was unturned followed by salpingectomy. Surrounding hematoma was evacuated. Left fallopian tube and both ovaries were normal. The left tube was also removed and specimens sent for histopathological evaluation. Histopathological evaluation showed congestion, hemorrhage and infiltration of right tube by inflammatory cells with presence of necrosis consistent with tubal torsion. The left fallopian tube showed presence of congestion with presence of a paratubal cyst.

The patient was discharged in stable condition with prophylactic antibiotic cover and advised follow up.

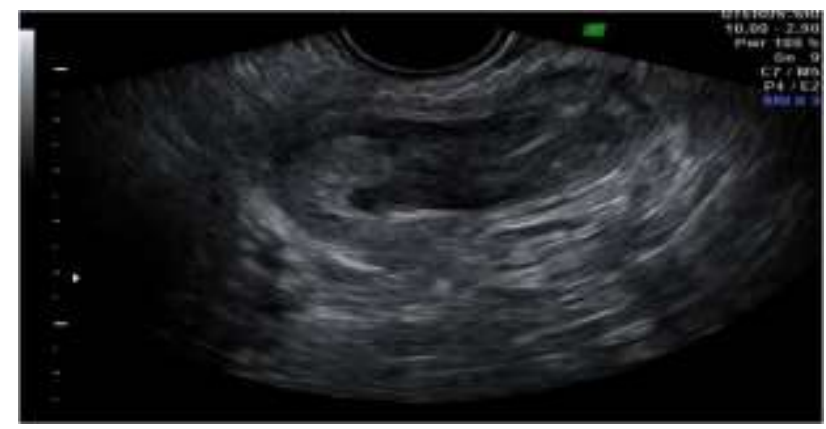

Figure 1: Dilated right fallopian tube with echogenic contents - suspicious for haematosalpinx.

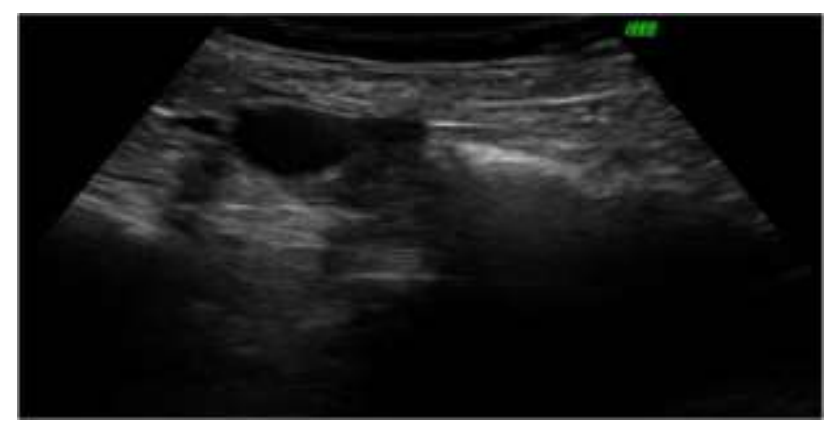

Figure 2: Normal appearing ipsilateral (right) ovary on transabdominal ultrasound.

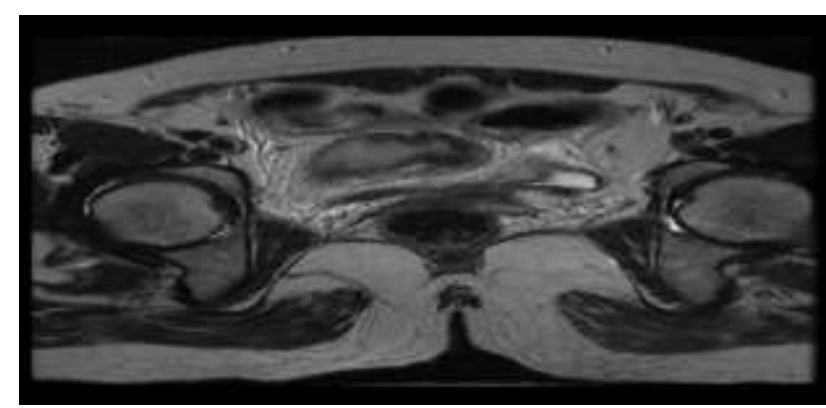

Figure 3: Axial T2WI: thick walled dilated tubular structure - dilated right fallopian tube.

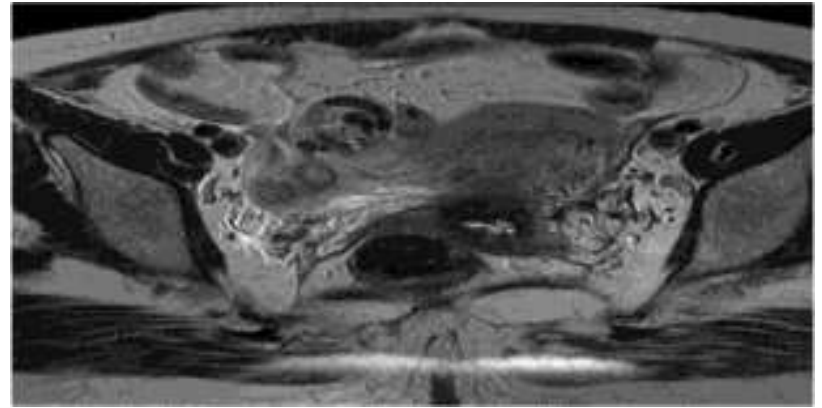

Figure 4: MRI whirlpool sign.

\section{DISCUSSION}

Isolated tubal torsion predominantly affects women of adolescent and reproductively active age group. While the exact cause is not certain, multiple possible contributory intrinsic and extrinsic factors have been postulated in literature. Intrinsic factors include hydrosalpinx, hematosalpinx, excessively long fallopian tube or its spiral course and prior surgical intervention (eg.tubal ligation). Extrinsic factors include adhesions, ovarian or paraovarian masses, pregnancy or tumoral enlargement of the uterus, venous congestion and trauma etc. ${ }^{4}$

The sequential events postulated in pathophysiology include initial mechanical obstruction of adenexal veins and lymphatics with unobstructed arterial flow. This results in congestion, tubal odema and enlargement with consequent tubal torsion ${ }^{5}$ Complications include tubal necrosis and gangrene with superadded infection and peritonitis. Ipsilateral ovary can also get irreversibly damaged due to ischemia. ${ }^{6}$

The nonspecific clinical presentation of tubal torsion makes clinical diagnosis challenging. Tubal torsion is included among the differential diagnosis of acute lower abdominal pain. Other conditions include ectopic pregnancy, ovarian torsion, ruptured ovarian cyst, pelvic inflammatory disease, acute appendicitis and other surgical emergencies. The laboratory findings are also nonspecific and do not pinpoint to the diagnosis. ${ }^{4}$ There is apparent predeliction and more frequent diagnosis of right sided tubal torsion. This can be partly attributed to the partial immobilization of left fallopian tube by sigmoid mesentery. Furthermore, frequent surgical management of right lower abdominal pain for management of suspected acute appendicitis attracts greater attention to this abnormality. The present case also had an isolated right tubal torsion. However, isolated tubal torsion occurring on left side has also been reported. ${ }^{7}$

Prompt diagnosis can potentially prevent irreversible changes of tubal necrosis and gangrene.

On ultrasound, presence of a dilated fallopian tube with normal appearing ipsilateral ovary should suggest the 
possibility of tubal torsion. ${ }^{8}$ Normal appearing uterus and ovaries with presence of pelvic free fluid, dilated thick walled tubular structure with echogenic walls, incomplete folds and internal echoes is the usual appearance of a torsed fallopian tube. ${ }^{9}$

Color Doppler shows no flow in the walls of hydrosalpinx. High resistance flow, reversal or absent flow in fallopian tube wall has been reported. But, confident spectral Doppler analysis of the tubal wall may be practically difficult. ${ }^{10}$ On color Doppler, the whirlpool sign is described. ${ }^{11}$ This is the result of tissue twisted around the central vascular pedicle and can be elicited by twisting the ultrasound probe back and forth along the axis of suspected torsion. In presence of normal appearing ovaries, whirlpool sign is considered specific for tubal torsion. The whirlpool mass in tubal torsion is relatively smaller and less obvious than ovarian torsion. That is why the whirlpool sign was probably not appreciable on sonography and Doppler in the present case.

Though CT features of tubal torsion are also described in literature, ${ }^{12}$ but $\mathrm{CT}$ is not considered to add further to the diagnosis beyond the information provided by ultrasonography. ${ }^{13}$

Aydin et al were the first to report the whirlpool sign on MRI in a case of tubal torsion. Direct visualization of this sign also clinched the preoperative imaging diagnosis of tubal torsion in our present case. MRI can also confirm the presence of hemorrhagic infarction by employing the fat suppressed T1 weighted and gradient (GRE) images. The sequence showed the presence of hematosalpinx in the present case. Presence of hemorrhagic infarction requires surgical removal of the tubes with no possibility of salvaging them, hence demarcating the surgical planning. While MRI whirlpool sign has been described for ovarian torsion in Indian literature, this is probably the first description of MRI whirlpool sign being employed (in the Indian subcontinent) for diagnosis of tubal torsion. ${ }^{14,15}$

This case reinforces the fact that in presence of whirlpool sign with normal appearing ovaries, there is a high index of radiological suspicion for tubal torsion which by itself is a rare entity.

\section{CONCLUSION}

This case reinforces the fact that in presence of whirlpool sign with normal appearing ovaries, there is a high index of radiological suspicion for tubal torsion which by itself is a rare entity.
Funding: No funding sources Conflict of interest: None declared Ethical approval: Not Required

\section{REFERENCES}

1. Hansen $\mathrm{OH}$. Isolated torsion of the Fallopian tube. Acta Obstet Gynecol Scand.1970;49(1):3-6.

2. Binkovitz L, Naprawa J, Binkovitz L. Cross sectional imaging of ovarian and isolated fallopian tube torsion. Accessed online from October 2015.

3. Bland Sutton J. Remarks on salpingitis and some of its effects. The Lancet. 1890;136(3509):1146-8.

4. Krissi H, Shalev J, Bar-Hava I, Langer R, Herman A, Kaplan B. Fallopian tube torsion: laparoscopic evaluation and treatment of a rare gynecological entity. J Am Board Fam Med. 2001;14(4):274-7.

5. Bernardus RE, Van der Slikke JW, Roex AJ, Dijkhuizen GH, Stolk JG. Torsion of the fallopian tube:some considerations on its etiology. Obstet Gynecol .1984;64(5):675-8.

6. Ghossain MA, Buy JN, Bazotetal M. CT in adnexal torsion with emphasis on tubal findings: correlation with US. J Comput Assist Tomogr. 1994;18(4):61925.

7. Wong SW, Suen SH, Lao T, Chung KH. Isolated fallopian tube torsion: a series of six cases. Acta Obstet Gynecol Scand. 2010;89(10):1354-6.

8. Shukla R. Isolated torsion of the hydrosalpinx: a rare presentation. Br J Radiol . 2004;77(921):784-6.

9. Sherer DM, Liberto L, Abramowicz JS, Woods JR Jr. Endovaginal sonographic features associated with isolated torsion of the fallopian tube. J Ultrasound Med. 1991;10(2):107-9.

10. Baumgartel P, Fleischer A, Cullinan J, Bluth R. Color Doppler sonography of tubal torsion. Ultrasound in Obstetrics and Gynecology. 1996;7(5):367-70.

11. Vijayaraghavan SB, Senthil S. Isolated torsion of the fallopian tube - the sonographic whirlpool sign. J Ultrasound Med. 2009;28(5):657-62.

12. Hiller N, Appelbaum L, Simanovsky N, Lev-Sagi A, Aharoni D, Sella T. CT features of adnexal torsion. Am J Roentgenol. 2007;189(1):124-9.

13. Kardakis S, Barranca A, Vitelli A, Amore I, Trento F, Caccia G. Isolated fallopian tube torsion. Case Rep Obstet Gynecol. 2013;2013:479698.

14. Aydin R, Bildircin D, Polat AV. Isolated torsion of the fallopian tube with hydrosalpinx mimicking a multiloculated ovarian cyst: whirlpool sign on preoperative sonography and MRI. J Clin Ultrasound. 2014;42(1):45-8.

15. Ghonge NP, Lall C, Aggarwal B, Bhargava P. The MRI whirlpool sign in the diagnosis of ovarian torsion. Radiol Case Rep. 2012;7(3).

Cite this article as: Thakral A, Malhotra R, Sobti N, Chandna A, Arora M. MRI diagnosis of tubal torsion: the whirlpool sign. Int J Reprod Contracept Obstet Gynecol 2016;5:237-9. 